\title{
The Effect of Retro-reflectivity and Reflectance of UK Number Plates on ANPR Performance
}

\author{
R.Gurney*, M.Rhead*, W.E.Martin*, S.Ramalingam*, N.Cohen ${ }^{\dagger}$ \\ * robert.gurney@herts.pnn.police.uk, michael.rhead@herts.pnn.police.uk,w.e.martin@herts.ac.uk, s.ramalingam@herts.ac.uk \\ School of Engineering \& Technology, University of Hertfordshire, Hatfield, Herts AL10 9AB, UK \\ † n.cohen@homeoffice.gsi.gov.uk, Home Office, Centre for Applied Science \& Technology, Sandridge, Herts. AL4 9HQ
}

Keywords: ANPR, Retro-reflectivity, Reflectance, Character Contrast, Spectrometer Analysis.

\begin{abstract}
The UK police service is one of the biggest users of ANPR technology in the world and over a number of years Police Forces have reported variances in ANPR performance based upon the retro-reflectivity properties of UK number plates. Laboratory and field-based research has been conducted with the aim of better understanding the effect of number plate component differences upon imaging systems used by law enforcement agencies for crime prevention and detection purposes. The retro reflective properties of number plates in the Infra-Red can depart significantly from visible light performance. The outcome of this work has implications for both the British Standard and for practical ANPR system design.
\end{abstract}

\section{Introduction}

Development of ANPR technology over the past few decades has led to the introduction of infra-red cameras that enable continuous capture of number plate data. Historically, there have been a number of problems for ANPR systems caused by a huge variety of lighting conditions, daytime, night-time, sunlight, backlight, headlights, and so on.

In practice, cameras for use in ANPR operate in the range of 500-850 nanometres (nm) spectrum. The latest cameras must be sensitive to the infrared part of the spectrum, to at least 850 nanometres. This is because the cheapest IR illuminators operate at $850 \mathrm{~nm}$ and are the most common type currently in use with ANPR systems. Most are also fitted with a filter to restrict the visible part of the spectrum, thus alleviating many of the lighting issues. Some manufacturers recommend that the best results can be obtained by setting the manual iris to fully open with the shutter speed set to $1 / 1000$ th second and by fitting an infrared source adjacent to the camera to provide additional illumination.
Other guidance recommends a small iris to prevent image blur caused by distance travelled whilst the shutter is open. Clearly one can see the important benefit of the retroreflective characteristics of number plates over other number plates. The illumination from the infra-red illuminator is reflected directly back to the camera and ONLY infrared light will be seen without any visible light or other reflections because of the filter. The resultant image is black with no detail except for the number plate. This does cause difficulty for some inexperienced observers who cannot understand why they cannot see the associated vehicle but to the interested observer it is a good indication as to whether or not the image has been obtained in the visible or infra-red part of the spectrum.

ANPR systems consist of a combination of hardware and software components, of which the Optical Character Recognition (OCR) is critical to correct interpretation of a vehicle registration mark. Once an image of the number plate has been captured, the OCR software will automatically convert it to usable code so it is important that there is sufficient contrast between the characters and the background. This is a key element of the recognition process and the importance of good contrast cannot be understated.

In the UK, number plate use and manufacture is governed by both legislation and the British Standard for number plates, known as BSAU 145d [1]. All UK number plates manufactured from 1973 onwards are required by law to be retro-reflective. This was a road safety initiative aimed at increasing the visibility of unlit vehicles parked on the road at night. There are vintage vehicles that are allowed to display non-retro reflective number plates but these are few in number. These plates present a particular challenge to ANPR systems as without reflective capability they cannot be illuminated in the traditional manner. i.e. low power pulsed illuminators.

One way to improve the accuracy of read data of all systems could be to use higher powered illuminators. Additionally, the use of narrow band filters may be helpful to match the wavelengths of the illuminator. This filter principle 
may also prove beneficial in eliminating other extraneous light sources such as street lights, vehicle headlights, sunlight, etc. The resulting image can be much clearer for optical character recognition (OCR) interpretation.

The reflectance measurement has not been reported in ANPR system performance analysis in practical applications anywhere in the world and this is the first research of its kind. Reflectivity and spectrum meters exist but field measurements are very difficult because of weather, lighting and other noise conditions. The aim of this research is to provide an assessment of the retro-reflectivity of real world number plates in use in the UK through spectrometer analysis of number plates with varying reflective properties.

This work follows on from previous research into the real world accuracy of Automatic Number Plate Recognition (ANPR) systems carried out over the past seven years on behalf of the Police Service nationally. [2] [3] [4].

The rest of the paper is organised as follows: Section 2 details the behaviour of reflected light and defines the related reflectivity parameters of measurement. Section 3 describes the experimental setup to measure reflectivity parameters of number plates and the testing process. Section 4 discusses the results of reflectivity analysis, inferences and recommendations suggested for improvement to the British Standard. Section 5 provides a conclusion and further work.

\section{Behaviour of Reflected Light}

When considering the reflective properties of number plates it is first helpful to look at the behaviour of light. The basic law of light is that the angle of incidence equals the angle of reflection. This is commonly termed "specular" reflection and most shiny surfaces are primarily specular in nature. Even transparent glass and, importantly for this investigation, most transparent acrylic number plate covers specularly reflect a portion of incoming light. [5] However, across the world and especially in the UK, due to legislation, number plates have a special characteristic; they are known as retro reflective. The reflective backing strip is covered in hundreds of tiny hemispheres which cause light to be reflected back to the source, utilising the same technology used in safety clothing and signs. The huge benefit of this is that no matter from which direction the light is shone, it always reflects back and makes them very visible. This principle can be seen in Fig 2.1. Away from the narrow angle retro reflection the reflectance of the surface closely resembles a non-specular reflection. This type of reflection is commonly known as diffuse reflection and is perhaps best illustrated in the reflectivity response of powders where there is no directional "shiny" component but it remains uniformly bright from any angle.

\subsection{Comparison with British Standard BSAU 145d}

The method of reflectivity assessment utilised in this experiment differs from the measurements conducted in the British Standard, BS AU 145d.

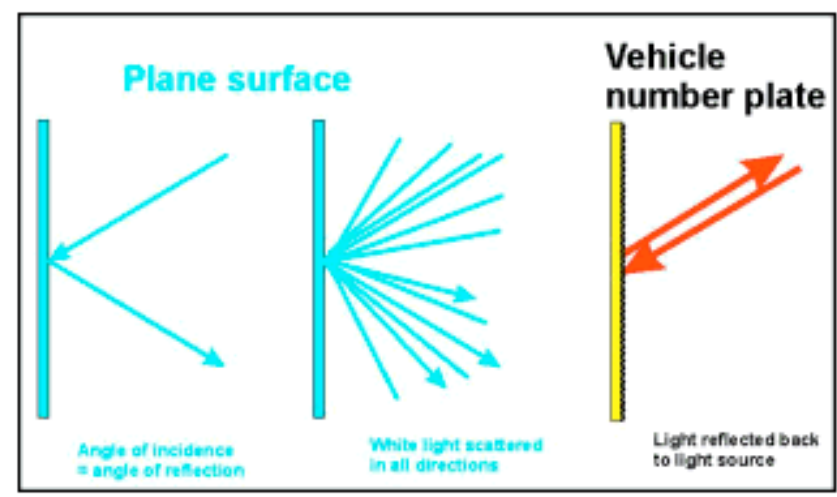

Fig 2.1 the behaviour of light on retro-reflective surfaces [6]

This research gives an insight into the reflective properties of British Number plates but it is important to note that the author has not measured R' as defined in the British Standard. The author has measured the reflectance of the material but the British Standard measurement combines the reflectance of the material with the properties of the retro-reflective layer which were specifically avoided by illuminating and measuring at bigger angles than the British Standard test.

The research conducted thus far suggests that separate tests of the IR retro-reflectivity of the retro layer may be necessary since it is not specified in the British Standard but the basic assumption is that the retro layer adds no 'colour' (variation with wavelength) to the measurements that have already been made. This might not be true for narrow band IR sources but that has never been measured.

\subsection{Converting between geometry based measurements}

This is a difficult task that should only be attempted when it is impossible to measure in the actual desired units. It is essential that one knows what each of the measurement geometries implicitly assumes before you can carry out a conversion.

In these reflectivity tests the authors have used radiometric measurement as opposed to the CIE method designated within the British Standard. The British Standard test was originally based purely on reflectivity in the visible spectrum and it naturally follows that the CIE method of measurement would have been internationally acceptable for that purpose. One must remember that although there have been a number of updates to the British Standard; none have taken account of developments in ANPR technology and the need for measurement in the infra-red spectrum.

Many Automatic Number Plate Recognition systems work within the near infra-red from a range of approximately $800 \mathrm{~nm}$ through to $1100 \mathrm{~nm}$. The basic reason behind this is that generally speaking typical silicon sensors are not sensitive above $1100 \mathrm{~nm}$. IR illuminators are cheap to produce where the illumination required falls within the near infra-red. This is a key factor in terms of the choice of wavelengths specified by manufacturers. Likewise it was a key factor in the choice of equipment used to measure reflectivity across the whole spectrum. 


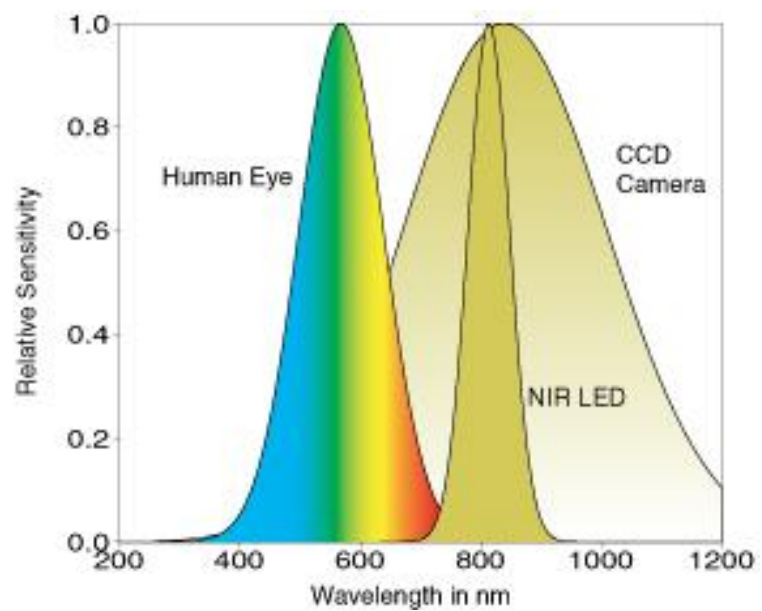

Fig 2.2 illustration of Human Eye sensitivity \& Camera CCD

In brief, radiometric units are purely physical quantities in contrast to photometric quantities that are based on the response of the human eye, as illustrated in Fig 2.2. It is therefore helpful to gain a better understanding of the two types of measurements before comparing our results to the British Standard.

Radiometry is perhaps best explained as "the measurement of electromagnetic radiation". In basic terms this means everything from $\mathrm{x}$-rays to microwaves and radio waves. Wavelengths range from less than a billionth of a meter for X-rays to greater than a meter for radio waves. In terms of the experimentation that we have conducted using optical systems we can limit this to light from the Ultraviolet part of the spectrum through to the Infrared spectrum.

A detailed list of radiometry and photometry properties and definitions are provided in LEDs magazine [7].

\section{Reflectivity Analysis: Experimental Setup}

Two separate experiments were conducted; initially field based followed by laboratory testing.

\subsection{Field Work}

The scope of the field work was to identify number plates that exhibited retro-reflectivity performance issues and to conduct a ground truth comparison. Two cameras were used, one of which captured images within the visible spectrum and another fitted with a high band filter effectively limiting light to above $830 \mathrm{~nm}$. This has the effect of identifying image capture problems within the IR spectrum used by ANPR systems

The Police found difficulties with Number Plates being read by ANPR systems operating within the Infra-Red spectrum. In order to establish the scale of this problem, a field study was initiated. This involved the specialist fitting of a high pass filter to a standard digital camera (Fig 2.3). This had the effect of only capturing images within the IR spectrum above $830 \mathrm{~nm}$.

The digital camera works by focussing an image onto the CCD. When a photograph is taken the image is captured by the CCD and then transferred to the camera's storage device such as an SD card. The electromagnetic wavelength range can be from 300 to $1,100 \mathrm{~nm}$. The human eye functions in the broad range 380 to $760 \mathrm{~nm}$ and this is known as the visible spectrum. Near infrared is usually considered to be in the range 750 to $2,500 \mathrm{~nm}$.

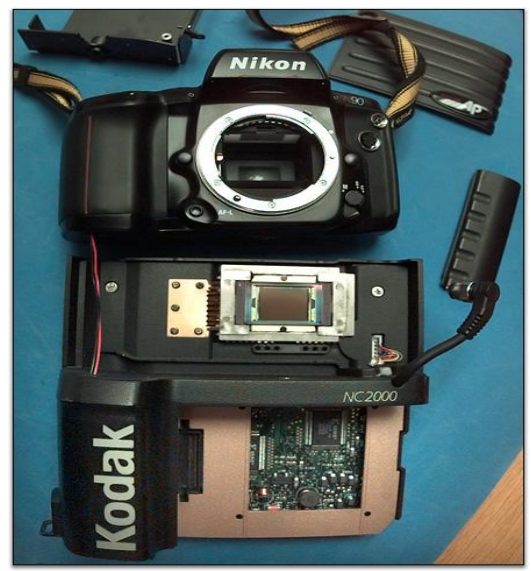

Fig 2.3 Exploded view of a standard digital SLR camera

A CCD in a digital camera will have a low pass filter (LPF) fitted across it (Fig.2.4). The CCD usually has one or more low-pass filters, which sit in front of the sensor. The purpose of a low pass filter is to limit the wavelengths at which it is able to operate. It will prevent wavelengths above a certain level from entering the CCD. This is done to remove light above a certain wavelength as it can cause undesirable effects if a normal white light photograph is required. $60 \%$ of the light provided by the sun is in the infrared range.

If the camera is to be used in the near infrared spectrum the low pass filter is usually removed and a high pass filter installed. The high pass filter only lets light above a certain wavelength enter the CCD. In the case of the specially adapted camera, an 830nm high pass filter was fitted and just to recap this means that only light at wavelengths above $830 \mathrm{~nm}$ reach the CCD to create an image.

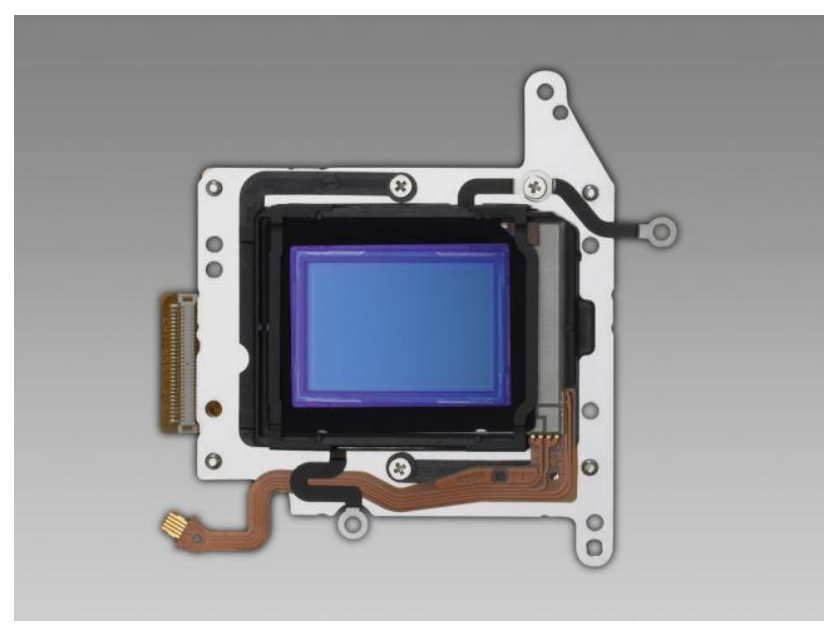

Fig.2.4 CCD camera with HPF fitted

The specially adapted camera was used to identify problematic number plates. Photographs were taken from the near side front passenger seat while driving very slowly. Once such a plate had been identified the researchers stopped 
moving and a standard camera was used to obtain an ambient light image of the problematic number plate, thereby providing ground truth. Immediate analysis of the captures was necessary to identify problematic plates.

\subsection{Lab-based Testing}

The scope of the lab test was to determine the reflectance of both background and character for each number plate sample. For the lab testing, a test data set of 43 number plate samples of varying ages drawn from a range of manufacturers were used $^{1}$. Laboratory time funding was limited and the authors initially examined number plates that were already in their possession. Future work will include a much greater database of number plates. The proposal was to measure illumination at:

1. Reference Optical Standard-A standard calibrated diffuse reflector (Spectralon [8]) was used for comparison.

2. Sample Observation points. Two samples per plate were taken with one from the large white or yellow area and another from within the black character area.

The number plate samples were measured for reflectance using a standard fiber optic spectrometer, an Ocean Optics model HR4000CG and a halogen light source. A standard calibrated diffuse reflector (Spectralon) was used for comparison and the measurements are expressed as the ratio of the light reflected from the standard reflector to the sample in percent [\%]. The relative reflectance of the white background and black numbers on the plates was measured at an incidence angle of 30 degrees.

\subsection{Findings on Reflectivity Coefficient}

Readings were taken across a wide spectrum but the researchers concentrated on readings obtained in the visible and near infrared from $400 \mathrm{~nm}$ to $1000 \mathrm{~nm}$. The lower and upper limits were chosen to avoid excessive noise at the extreme ends of the spectrum. Optimum reflectivity is illustrated by good contrast between the characters and background, both in the visible and invisible spectrum.

\section{Discussions and Recommendations}

The results of the experiment ate plotted in Figs.3.1-3.2. A summary is made as follows:

Variances in performance were discovered between front (white) and rear (yellow) number plates, with the rear plates generally performing worse than the front plates. This is supportive of many field installations that capture front facing plates only. Whilst there are a number of reasons for front capture preference, ranging from over-view photograph opportunities to physical site limitations, better capture rates are observed with white (front) number plates.

\footnotetext{
${ }^{1}$ All tests were conducted in the Polarimetry laboratory within the School of Physics, Astronomy and Mathematics at the University of Hertfordshire.
}

The experimental sample contained a variety of plate types and included some with clear acrylic type coverings that are designed to protect the printed characters. The authors' original field trials suggested that the covering layer may cause attenuation at certain wavelengths and there was a concern that this could adversely affect performance. This is borne out by the laboratory testing where a peak and trough effect occurs between $800 \mathrm{~nm}$ and $950 \mathrm{~nm}$ in all plates with acrylic covers.

Some manufacturers have produced number plates with a very thin plastic film covering. In the field, performance issues have been observed where the thin covering has been worn or torn away - often removing the characters beneath them. In the laboratory, the authors observed similar reflective performance to that of the basic backing film strips, i.e. higher reflectivity than those with acrylic type coverings.

In order to best illustrate the generic behaviour of retroreflective number plates, two scatter graphs have been created, Fig 3.1 \& Fig 3.2. Each small bar in the graphs is the reflectivity of an individual number plate. The upper clusters are the background measurements and the lower clusters are the number reflectivities. Although a wide range of readings were obtained across the spectrum, the authors have chosen to highlight two specific wavelengths to assist the reader to understand real world characteristics. The first wavelength chosen is $550 \mathrm{~nm}$ as this represents the optimum wavelength within the visible spectrum. The second wavelength chosen is $850 \mathrm{~nm}$ as this represents the most common wavelength utilised in ANPR systems.

Fig 3.1 illustrates the generic retro-reflective characteristics of "White" front number plates whilst the second, Fig 3.2 demonstrates those of "yellow" rear number plates. If one examines the patterns displayed by white or "front" number plates, one can clearly see the contrast in the form of a pronounced gap between the backing layer and the characters.

At $850 \mathrm{~nm}$ one can see the drop in reflectivity of the white number plate compared to that at $550 \mathrm{~nm}$. Under infrared conditions and in this case at $850 \mathrm{NM}$, contrast can be well displayed for many of the front number plates.

In many cases for the front number plates the contrast is clearly defined on the by a visible gap between the backing and the characters. Experience dictates that well set up cameras should not have any particular difficulty reading any of the number plates providing there is good contrast.

Turning to the yellow "rear" number plate illustrated by Fig 3.2 we can see that there are some significant issues within the visible spectrum. Although there is a level of contrast between the backing and the characters, it is in fact virtually non-existent at the optimum visible wavelength.

Conversely however within the infrared spectrum one can see that the contrast is in fact very good.

The practical effect is that witnesses and others who need to be able to read and comprehend what is shown on a number plate may not find it very easy within the visible spectrum if a particular type of number plate is displayed. 


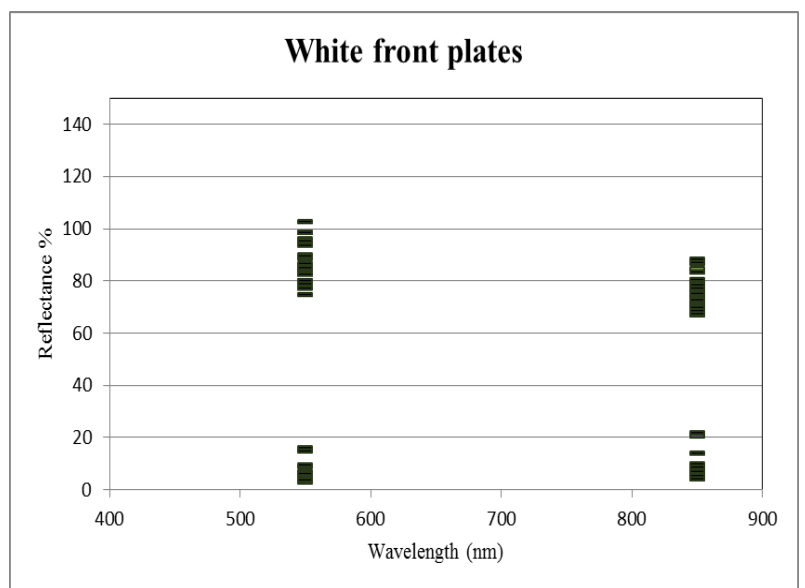

Fig.3.1 Retro-reflective characteristics of "White" front number plates

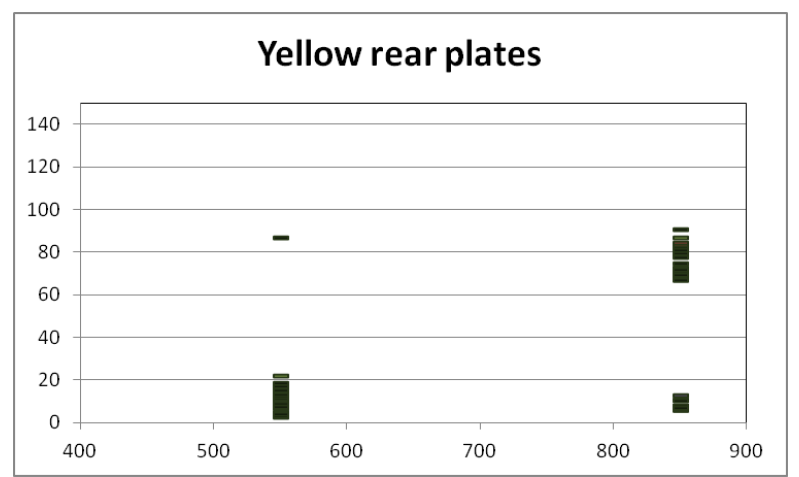

Fig.3.2 Retro-reflective characteristics of "Yellow" rear plates

This testing has shown that a variety of reflective backing layers have been used across the board with varying levels of performance demonstrated in the findings above and below.

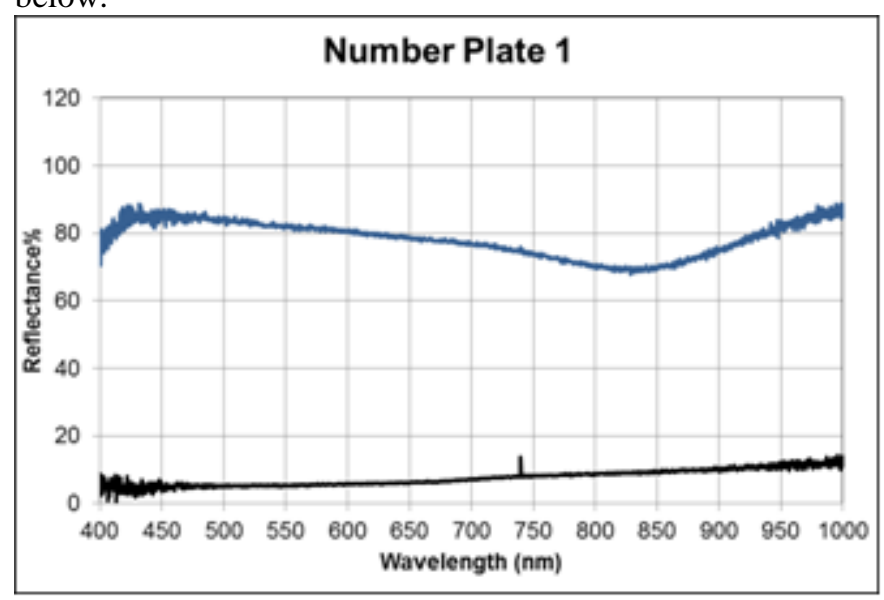

Fig.3.3 Standard White NP

Fig 3.3 illustrates a typical measurement exhibited by a standard front number plate with mono black characters on a white background. The reflective backing on this plate had what one might refer to as "regular" beads. In this case we see the black lettering at the base of the graph with the reflective white backing represented by the upper blue line. One can see good contrast between the two. Note the dip in backing reflectance around $850 \mathrm{~nm}$ - this is common in most backing strips that the researchers have examined.

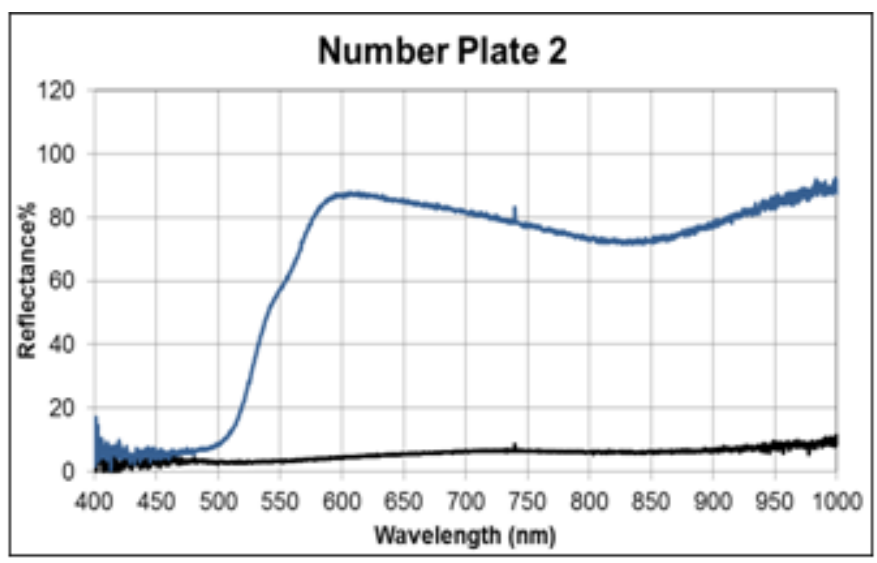

Fig 3.4 Standard Yellow Number Plate

Fig 3.4 is a typical example of a rear number plate with black characters on a yellow reflective backing strip.

Note there is little reflectance below 500nm (blue-green) and thus appears yellow in white light. From this point onwards the readings increase in a linear fashion up to around 600nm at which point we see good contrast against the black lettering. ANPR systems will perform much better with good contrast so it is essential that the correlation between the characters and backing remains relatively constant in the visible and infra-red where the cameras are most sensitive.

\section{Conclusion and Further Work}

In this paper, the authors set about performing ground truth analysis and field study on retro-reflectivity analysis. These were successfully carried out with a small set of plates but sufficient enough to draw the necessary conclusions. The result of the experiments revealed the various contributing errors in capture and read rates and it has also revealed that their impacts varied as expected.

The authors organised a technical workshop for ANPR manufacturers that took place at the University of Hertfordshire on 29 May 2013. The results of this study formed the basis of reflectivity discussions at that industry workshop that was designed to assist in the formulation of recommendations around number plate construction and relevant legislation.

A number of changes have been made by number plate manufacturers to their own number plate construction processes and the authors have established that a variety of component part changes have taken place. In particular, this testing has shown that a variety of reflective backing layers have been used across the board with varying levels of performance demonstrated in the findings above. It is important to note that number plates that appear outwardly identical to the human eye have been built using differing component parts, yet no British Standard testing has been conducted to assess the effect of these changes on performance. Internationally, the last time that any research 
was conducted in this field was by Post, Pollock and Mortimer in 1974 [9] and Israelson \& Coleman in 1980 [10]. The author recommends a review of the British Standard (BS AU 145d) with a view to amendment to accommodate a number of changes as a result of wider joint research but in particular in relation to reflectivity testing.

The CIE is internationally renowned as the standard for light measurement within the visible spectrum and the authors do not seek to replace that standard. But it is clear that the CIE measurement is inadequate for measurements in the near infra-red and the author recommends further research to identify a suitable method.

Further research into ANPR Camera Settings is reported by the authors in "The effect of ANPR Camera Settings on System Performance" [11]

\section{Acknowledgements}

This research has only been made possible through the assistance of staff at the Centre for Atmospheric and Instrumentation Research at the University of Hertfordshire where specialist laboratory facilities and technical support were made available by the kind agreement of the Director of Research for the Science and Technology Research Institute, Professor Paul Kaye.

Acknowledgments are also very much due to Chair of the ACPO ANPR Countermeasures Group, Detective Superintendent Paul Ealham for enabling this research to be undertaken.

\section{References}

[1] BS AU 145d: 1998, Specification for retro-reflecting number plates, BSI, 15 January 1998, ISBN 0580289850.

[2] M. Rhead, R. Gurney, N. Cohen, S. Ramalingam, Accuracy of Automatic Number Plate Recognition (ANPR) and Real World UK Number Plate Problems, IEEE International Conference on Security Technology, 15-18 Oct. 2012, Boston, Massachusetts, USA.

[ 3] R. Gurney, M. Rhead S. Ramalingam, N. Cohen, Working Towards an International Automatic Number Plate Recognition (ANPR) Standard - An Initial Investigation into the UK Standard, IEEE International Conference on Security Technology, 15-18 Oct. 2012, Boston, Massachusetts, USA.

[4] M. Rhead, R. Gurney, S. Ramalingam, N. Cohen, Impact of UK Number Plates on the Accuracy of Automatic Number Plate Recognition (ANPR), Journal of Information Assurance and Security Letters, http://www.mirlabs.net/iasl/, accepted and revised submitted, Aug. 2012.[5] H. Roy, "Wavelength considerations", http://web.archive.org/web/20071028072110/http://info.tuwie n.ac.at/iflt/safety/section1/1_1_1.htm , accessed 16th March 2013.

[6] The Light Measurement Handbook - Alex Ryer, International Light (1997)
[7] LEDs magazine, Radiometric and Photometric Terms, January 2004 http://ledsmagazine.com/features/1/1/11

[8] Labsphere Spectralon, 1986

[9] Post, Pollock \& Mortimer, A study of Reflectorized materials for licence plates. Michigan, USA. $28^{\text {th }}$ June 1974

[10] C.E. Israelson \& R.V. Coleman, Evaluation of Legibility of Reflectorized License Plates, Utah, June 1980

[11] R.Gurney, M.Rhead, V.Lyons, S.Ramalingam, "The effect of ANPR Camera Settings on System Performance" IET $5^{\text {th }}$ International Conference on Imaging for Crime Prevention and Detection ICDP-13, Vision and Imaging Network, 16-17 ${ }^{\text {th }}$ December 2013, London, UK. 